\title{
Uso de neurolépticos atípicos en esquizofrénicos consumidores de cannabis
}

\author{
Núñez Domínguez, L.A.
}

Clínica San Francisco Javier. Médico Psiquiatra.

Enviar correspondencia a:

Dr. Luis Alonso Núñez Domínguez. Clínica San Francisco Javier. Avenida Baja Navarra, 52. 31002 Pamplona. Tel. 948231600.

\section{RESUMEN}

El alto número de artículos de investigación publicados en torno a la patología dual refleja el creciente interés por este novedoso campo de la psiquiatría, lo cual no es de extrañar, dados los altos índices de prevalencia de consumo de tóxicos entre los pacientes afectos de patología mental. La mayoría de dichos trabajos contemplan un tratamiento integral, basado en la aplicación de técnicas psicológicas combinadas con un tratamiento farmacológico, pero son escasas las referencias en torno a este último punto.

En el caso del consumo de cannabis asociado a esquizofrenia existen referencias en torno a un mayor beneficio del uso de neurolépticos atípicos. El objetivo del presente estudio es verificar si el uso de tales fármacos es más útil para el manejo de este tipo de pacientes.

Material y método: Se comparan tres grupos de esquizofrénicos paranoides, dos de ellos con consumo de cannabis, uno de ellos tratado con neurolépticos clásicos y el otro con atípicos. A los 3 grupos se les realiza un seguimiento durante un año

Resultados: Se observa una reducción en la frecuencia del consumo en el grupo tratado con atípicos y una mayor conciencia de enfermedad, manifestado en un menor número de ingresos involuntarios

Conclusiones: Los resultados sugieren que el uso de neurolépticos atípicos es más beneficioso para el manejo de pacientes esquizofrénicos consumidores de cannabis

Palabras clave: Patología dual. Cannabis. Esquizofrenia. Neurolépticos atípicos

\section{ABSTRACT}

The increasing research about comorbidity shows the growing importance of this topic in the current development of psychiatry. The mayority of these papers discuss the importance of psychological treatment in these kind of patients, but there are a few number dealing with the pharmacological treatment.

There are some references on the benefit of the atypical neuroleptics in the treatment of schizophrenic patients using cannabis. The aim of the present paper is trying to verify this hypothesis.

Material and Methods: Three groups of patients with paranoid schizophrenia, two of them cannabis users, were included in this study. We made a one year follow-up after treatment with a classic (group ED) or an atypical (group AT) neuroleptic treatment

Results: Group AT shows a lower frequency of cannabis use and a greater insight.

Conclusions: Atypical neuroleptics could be useful in the management of schizophrenic patients with current cannabis use.

Key words: Comorbidity. Cannabis. Schizophrenia. Atypical neuroleptics.

\section{INTRODUCCIÓN}

$\mathbf{L}$ a publicación por parte de NIDA del libro Principles of drug addiction treatment: A researchbased guide (1999) puede servir de botón de muestra de que la patología dual está adquiriendo una importancia creciente en los últimos años, debido al importante número de pacientes psiquiátricos que consumen drogas por uno u otro motivo. Las cifras de prevalencia situan entre un 30 y un $80 \%$ el número de pacientes con patología mental que consumen sustancias tóxicas (Regier y cols., 1990).

Después del alcohol y el tabaco, las dos sustancias más utilizadas entre los sujetos con esquizofrenia, el cannabis aparece como la sustancia ilegal más consumida, sobre todo entre los pacientes esquizofrénicos, en torno al $60 \%$ de sujetos esquizofrénicos serían consumidores habituales de cannabis (Regier y cols., 
al., 1990), aunque con baja detección del mismo (Noguera Ormazábal y cols., 1993), a pesar de nuevas fórmulas de detección de consumo como el SCID-P (Albanese y cols., 1994). Entre las hipótesis que intentan explicar este alto consumo podríamos citar la de la automedicación (Khantzian, 1985): búsqueda de superación de las dificultades motivadas por el deterioro esquizofrénico, con mayor integración en la sociedad (Camí, 1988), deseos de activación (Muesser et al., 1992) o de relajación (Linszen et al., 1994), o de eliminar los efectos secundarios de la medicación antipsicótica (Knudsen y Vilmar, 1984); o la hipótesis de "vulnerability-stress-coping" de Hambrecht (1999), más centrada en el consumo de cannabis. Este autor hipotetiza que el consumo de cannabis provoca un aumento de estrés psicológico, lo cual puede provocar en le paciente un aumento de la vulnerabilidad hacia la psicosis y conducirle a un estado psicótico, el cual pretende aliviar manteniendo el consumo de la sustancia.

Dadas las consecuencias que el consumo provoca en este tipo de pacientes, como inicio precoz de la enfermedad, (Shaner y cols., 1993), aumento de la sintomatología positiva (Peralta y Cuesta, 1992; Allebeck et al., 1993), mayor número de ingresos (Stone y cols., 1993), mayor refractariedad al tratamiento (Bowers et al., 1990) y peor cumplimiento terapeútico (Dixon et al., 1990), en ocasiones motivado por la falta de conciencia de enfermedad (Pristach y Smith, 1990), todos ellos indicadores de mala evolución, nos encontramos en una fase de búsqueda de tratamientos eficaces para este grupo de pacientes, no sólo desde el punto de vista psicosocial, sino también farmacológico.

Son escasas las referencias al tipo de tratamiento farmacológico en este tipo de pacientes. Siris y cols. (1990) recomiendan el uso de dosis baja de imipramina para el episodio depresivo post-psicótico en pacientes esquizofrénicos consumidores de cannabis. En lo referente al tratamiento con los neurolépticos clásicos, Pristach y Smith (1990) refieren peor cumplimiento terapeútico, en parte debido a los efectos secundarios que provocan; Knudsen y Vilmar (1984) describen una mayor refractariedad al tratamiento neuroléptico.

Los neurolépticos atípicos han mostrado su utilidad en el manejo de este tipo de pacientes: Buckley y cols. (1994) han descrito una mejor respuesta al tratamiento con clozapina que con neurolépticos clásicos (por ej., haloperidol) en un grupo de esquizofrénicos con consumo de drogas. Yovell y Opler (1994) y Buckley (1998) consideran que la clozapina puede ser útil para reducir el consumo de alcohol, tabaco y cocaína. La olanzapina, en estudios en ratas, atenúa los efectos reforzadores de la cocaína (Meil y Schechter, 1997) y es taan eficaz como el haloperidol, con menos efectos secundarios, en el tratamiento de las psicosis cannábica (Berk y cols., 1999). En cuanto a la risperidona, ésta ha mostrado capacidad para reducir el deseo de cocaína durante la retirada de la misma (Smelson y cols, 1997) y ha mostrado ser útil para conseguir la reducción de los opiáceos ilegales en esquizofrénicos en tratamiento con metadona (Casas y cols., 1997) y para controlar un episodio de psicosis con metanfetamina (Misra y Kofoed, 1997)

En el presente estudio se comparan tres muestras de pacientes esquizofrénicos, dos de ellas con consumo de cannabis y una tratada sólo con neurolépticos atípicos, y comparamos los datos obtenidos en el seguimiento durante un año con el fin de valorar si dicho tratamiento produce mejores efectos.

\section{MATERIALY MÉTODO}

La Red de Salud Mental Pública de la Comunidad Autónoma de Navarra está compuesta por ocho Centros de Salud Mental, dos Hospitales de Día Psiquiátricos, un Centro de Día Psiquiátrico y dos Unidades de Hosptilación Psiquiátrica, a las que podemos unir tres Unidades de Hospitalización de carácter privado con conciertos para admitir pacientes de la Red, si el caso lo precisa. De entre los pacientes de dicha red se seleccionaron para este estudio aquellos sujetos que cumplieran los siguientes requisitos:

1- Pacientes que habían sido ingresados, al menos una vez, en cualquier Unidad de Hospitalización Psiquiátrica de la Red de Salud Mental de Navarra.

2- Pacientes con diagnóstico de trastorno esquizofrénico de tipo paranoide (Criterios DSM-IV).

3- Pacientes con el mismo diagnóstico previo $y$, como segundo diagnóstico, Trastorno por abuso de cannabis (Criterios DSM-IV)

Los pacientes que pertenecían a este último grupo se dividieron en dos grupos en función del tipo de neurolépticos que iban a recibir (clásicos o atípicos).

Con los pacientes que cumplían con los criterios de selección de la muestra, se formaron tres grupos de pacientes:

1- Grupo E: sujetos con diagnóstico de Trastorno esquizofrénico de tipo paranoide, sin consumo de drogas, y tratados con neurolépticos atípicos (clozapina, risperidona, olanzapina) durante el año 1999

2- Grupo AT: Sujetos esquizofrénicos con consumo de cannabis como substancia principal y tratados solamente con neurolépticos atípicos a partir de enero de 1999.

3- Grupo ED: sujetos esquizofrénicos con consumo de cannabis como substancia principal, y tratados solamente con neurolépticos clásicos (sobre todo, haloperidol) a partir de enero de 1999. 
Durante la entrevista de evaluación del paciente, se llevó a cabo la recogida de información referida a datos sociodemográficos generales (edad, sexo, antecedentes psiquiátricos familiares, etc.), historia de la enfermedad (edad de inicio, número y tipo de ingresos, etc. ), así como de la historia del consumo de drogas. La información obtenida se complementó y verificó con otra entrevista con familiares del paciente.

Posteriormente se llevó a cabo un estudio observacional, prospectivo y comparativo: el seguimiento se realizó durante un periodo de un año (año 1999), en el que se llevaron a cabo entrevistas de evaluación cada mes para valorar el consumo de sustancias y la evolución del paciente. El control del consumo se llevó a cabo con la realización de análisis de orina la menos una vez al mes. Aquellos pacientes a los cuales no fue posible realizar un seguimiento completo durante el año 1999 fueron eliminados del estudio, lo cual sucedió en 5 casos en el grupo E, 2 en el ED y 1 en el AT. Al final del periodo de seguimiento, los grupos estaban compuestos por el siguiente número de sujetos: grupo E 30 pacientes; grupo ED 16 pacientes, y grupo AT 20 pacientes.

La asignación del tipo de neuroléptico se llevó a cabo al azar. Las dosis de neuroléptico atípicos fueron de entre 100 y 300 mg al día de clozapina, entre 2 y $8 \mathrm{mg} / \mathrm{día}$ de risperidona, y entre 5 y $15 \mathrm{mg} / \mathrm{día}$ de olanzapina, en función de la sintomatología que presentaba cada caso.

Los datos obtenidos se compararon estadísticamente con la prueba de t de Student para comparación de medias y Chi cuadrado para comparación de porcentajes.

\section{RESULTADOS}

Los sujetos del grupo E son significativamente mayores en edad, con una mayor frecuencia de mujeres y mayor número de antecedentes familiares de esquizofrenia

\begin{tabular}{|ccccccc}
\hline \multicolumn{7}{c}{ TABLA 1. DATOS GENERALES } \\
\hline \multicolumn{7}{c}{ GRUPOS } \\
\hline E & ED & AT & E/ED & E/AT & ED/AT \\
\hline SEXO H & 17 & 13 & 20 & 0,0006 & NS & NS \\
M & 13 & 3 & 0 & & & \\
EDAD MEDIA & $41+11,8$ & $30+6,0$ & $29,1+4,2$ & 0,0005 & NS & NS \\
Rango & $19-66$ & $21-45$ & $22-36$ & & & \\
ANTECEDENTES PSIQUIÁTRICOS FAMILIARES & & & \\
NO & 12 & 10 & 11 & & & \\
ESQUIZOFR & 12 & 0 & 5 & & & \\
DEPRESIÓN & 2 & 2 & 0 & & & \\
ALCOHOL & 3 & 1 & 3 & & & \\
\hline
\end{tabular}

En lo referente a la historia de la enfermedad, los pacientes del grupo E presentaron los primeros síntomas a una edad significativamente mayor que los sujetos del grupo AT (con una tendencia hacia la significación con respecto al grupo ED), y la duración de la enfermedad en meses es significativamente mayor que la media de los otros dos grupos. Asímismo la edad media del primer ingreso es significativamente menor en los sujetos de los dos grupos de consumidores. No se observan diferencias en el número en el tipo de ingreso (voluntario o involuntario) con los otros dos grupos

\begin{tabular}{|c|c|c|c|c|c|c|}
\hline \multicolumn{7}{|c|}{ TABLA 2. HISTORIA DE LA ENFERMEDAD } \\
\hline & \multicolumn{3}{|c|}{ GRUPOS } & \multicolumn{3}{|c|}{$\mathbf{P}$} \\
\hline & E & ED & AT & E/ED & E/AT & ED/AT \\
\hline EDAD $1^{\circ}$ SINTOMAS (años) & $25,4+9$ & $21,1+2,8$ & $19,1+2,0$ & 0,09 & 0,015 & NS \\
\hline DURACIÓN ENFERMEDAD (meses) & $184,3+111$ & 101,6 & $112,4+73$ & 0,0078 & 0,014 & NS \\
\hline EDAD $1^{\circ}$ INGRESO (años) & $28,2+10,2$ & $22,5+2,5$ & $21,8+2,8$ & 0,036 & 0,008 & NS \\
\hline $\mathrm{N}^{\circ}$ INGRESOS & $5,2+4,6$ & $3,68+2,9$ & 6,85 & NS & NS & NS \\
\hline \multicolumn{7}{|l|}{ TIPO INGRESO } \\
\hline Judicial & 15 & 8 & 15 & NS & NS & 0,059 \\
\hline Voluntario & 15 & 8 & 5 & & & \\
\hline
\end{tabular}

En lo referente a la historia del consumo de drogas en los grupos ED y AT, observamos que en ambos grupos la mayoría de los sujetos comenzaron el consumo de sustancias antes del inicio de la enfermedad (14 en el grupo ED y 19 en el AT), y a una edad similar. Con la misma sustancia han mantenido una frecuencia de consumo parecida hasta el año previo al estudio y la duración media del consumo es similar.

TABLA 3. HISTORIA DEL CONSUMO DE DROGAS

\begin{tabular}{|c|c|c|c|}
\hline & \multicolumn{2}{|c|}{ GRUPOS } & \multirow{2}{*}{$\frac{P}{E D / A T}$} \\
\hline & ED & AT & \\
\hline EDAD INICIO CONSUMO (años) & $16,5+1,9$ & $17+2,4$ & NS \\
\hline \multicolumn{4}{|l|}{ DROGA INICIO } \\
\hline CANNABIS & 16 & 20 & NS \\
\hline \multicolumn{4}{|c|}{ INICIO CONSUMO DE DROGAS PREVIO A ENFERMEDAD } \\
\hline sí & 14 & 19 & \multirow{3}{*}{ NS } \\
\hline NO & 2 & 0 & \\
\hline A la vez & 0 & 1 & \\
\hline \multicolumn{4}{|l|}{ FRECUENCIA CONSUMO PREVIO } \\
\hline Varios/semana & 1 & 3 & \multirow{4}{*}{ NS } \\
\hline Fin de semana & 3 & 3 & \\
\hline 1/día & 5 & 5 & \\
\hline Varias/día & 7 & 9 & \\
\hline MESES DE CONSUMO & $133,5+67,7$ & $122,05+68,3$ & 3 NS \\
\hline
\end{tabular}


Las diferencias se observan durante el año de seguimiento: en ambos grupos disminuye la frecuencia del consumo, algunos sujetos del grupo ED han cambiado de sustancia para su uso habitual (cocaína y anfetamina). En el grupo AT se observa abandono del consumo en 4 casos y una mayor proporción de ingresos voluntarios en el último año.

\section{TABLA 4. DATOS AÑO 1999}

\begin{tabular}{|c|c|c|c|}
\hline & \multicolumn{2}{|c|}{ GRUPOS } & \multirow[t]{2}{*}{$\mathbf{P}$} \\
\hline & ED & AT & \\
\hline \multicolumn{4}{|l|}{ TIPO DROGA CONSUMIDA } \\
\hline CANNABIS & 13 & 20 & \\
\hline ANFETAMINA & 1 & 0 & NS \\
\hline COCAÍNA & 2 & 0 & \\
\hline \multicolumn{4}{|l|}{ FRECUENCIA CONSUMO DROGA } \\
\hline NO & 0 & 3 & \\
\hline Menos 1/semana & 0 & 1 & \\
\hline 1/semana & 0 & 0 & \\
\hline Varios/semana & 4 & 3 & NS \\
\hline Fin de semana & 4 & 2 & \\
\hline 1/día & 2 & 6 & \\
\hline Varios/días & 6 & 5 & \\
\hline INGRESOS AÑO 99 & $0,36+0,5$ & $0,65+1,1$ & NS \\
\hline \multicolumn{4}{|l|}{ TIPO INGRESO } \\
\hline NO & 11 & 13 & \\
\hline Judicial & 0 & 3 & 0,03 \\
\hline Voluntario & 4 & 4 & \\
\hline \multicolumn{4}{|c|}{ FRECUENCIA DE CONSUMO (RESPECTO AL PERIODO PREVIO) } \\
\hline Menor & 7 & 9 & \\
\hline Mayor & 5 & 1 & 0,022 \\
\hline Igual & 4 & 10 & \\
\hline
\end{tabular}

\section{DISCUSIÓN}

Los datos encontrados en el presente trabajo coinciden con los hallados en otros estudios similares: edad más precoz de inicio de enfermedad en los consumidores (Muesser y cols., 1990), mayor número de ingresos (Negrete y cols., 1986) (los grupos ED y AT presentan similar número de ingresos que el grupo $E$, con una duración menor de enfermedad), y mayores trastornos de conducta (mayor número de ingresos judiciales en el grupo AT) (Martínez Arévalo y cols., 1992). Los dos grupos con diagnóstico doble muestran un alto perfil de gravedad, quizás motivado por el hecho de haber seleccionado pacientes que hayan sido ingresados al menos una vez. En este sentido los sujetos del grupo AT podrían ser considerados muy graves (alto número de ingresos y de carácter involuntario). Destacar que los grupos ED y AT muestran una mayoría significativa de varones

También se observa que la mayoría de los consumidores comenzaron el uso de cannabis previamente al inicio de la esquizofrenia, factor de riesgo para la esquizofrenia (Martínez Arévalo y cols., 1992) y de peor evolución a largo plazo para los esquizofrénicos
(Núñez Domínguez, 1997). Este hecho podría aportar consistencia a la teoría ya expuesta de vulnerabilitystress -coping de Hambrecht (1999) como motivo para el consumo de cannabis entre los esquizofrénicos. También podríamos interpretar este hallazgo como sustento a la teoría del papel etiológico del cannabis en la esquizofrenia (Andreasson y cols., 1987; Emrich y cols., 1997; Núñez Domínguez, 1998; Schneider y cols., 1998): 5 sujetos del grupo AT presentan antecedentes familiares psiquiátricos de esquizofrenia, factor de alto riesgo para que los consumidores de cannabis presenten trastornos psicóticos. Pero el diseño del estudio no permite sacar más conclusiones en este sentido.

Hemos observado una disminución en la frecuencia del consumo en el último año en relación al consumo previo, de manera casi significativa $(p=0,08)$ entre los que han recibido neurolépticos atípicos, que presentan una frecuencia significativamente menor $(p=0,022)$ de consumo que los sujetos del grupo ED, lo que confirma los datos obtenidos por Buckley y cols (1994) que describen una mejor respuesta a la clozapina en un grupo de esquizofrénicos con consumo de drogas. En un estudio llevado a cabo por Casas y cols (1999) se observó una disminución del consumo de opiáceos entre sujetos esquizofrénicos tratados con risperidona. Este hecho podría explicarse de diversas formas: los neurolépticos atípicos no provocan en los esquizofrénicos tantos efectos secundarios molestos, lo que evitaría el uso de sustancias como forma de aliviar dichos efectos. O bien los sujetos tratados con estos fármacos perciben un mayor control de su enfermedad, sin que precisen otros remedios.

Se ha utilizado el número de ingresos y la voluntariedad de los mismos como medida más objetiva de conciencia de enfermedad, dado que las respuestas de los sujetos en torno a tipo de sustancia y frecuencia de consumo de tóxicos no suele mostrar altos índices de fiabilidad en los estudios llevados a cabo con toxicómanos. (Hindin y cols., 1994). A su vez, la conciencia de enfermedad puede ser empleada como criterio de gravedad, dado que, a mayor conciencia de enfermedad, mayor cumplimiento terapeútico (Lin y cols., 1979).

El autor ha encontrado una menor frecuencia en la proporción de ingresos involuntarios en el grupo AT $(p=0,018)$ durante el periodo de seguimiento, que correlaciona de manera casi significativa con la frecuencia de consumo en el último año $(p=0,09)$, lo que podría ser interpretado como una mayor conciencia de enfermedad (mayor "insight"). Dicho parámetro ha sido correlacionado con diversos aspectos de la enfermedad mental en los esquizofrénicos, y donde la relación ha demostrado mayor robustez es entre el cumplimiento terapeútico y la presencia de conciencia de enfermedad (Ghaemi y Pope, 1994). 
Young y cols. (1993) han relacionado el deterioro de las funciones en el lóbulo frontal con un menor insight de los pacientes. Struve y cols. (1998) obtienen un menor rendimiento entre sujetos consumidores de cannabis tras la administración de una batería de tests neuropsicológicos que estudian el funcionamiento del lóbulo frontal (test de Wisconsin, Test de Categorías, Test de Stroop y el test de Weschler). Podríamos deducir que el consumo mantenido en el tiempo y con alta frecuencia de cannabis provoca una alteración en el lóbulo frontal, lo que provocaría una disminución del insight, que podría producir un menor cumplimiento terapeútico; el consumo de cannabis produciría un aumento de la sintomatología, y todo ello conduciría a la necesidad de un ingreso forzado del sujeto, con el fin de controlar su enfermedad. David y cols. (1992) encuentran que los sujetos ingresados de manera involuntaria presentan un menor insight y más sintomatología que los ingresados de manera voluntaria. McEvoy y cols. (1989) describen un mayor insight en los pacientes que son hospitalizados con más frecuencia, sugiriendo que el paciente admite más fácilmente la necesidad del ingreso cuando su estado lo precisa.

Van Putten y cols. (1976) describen un menor insight en los esquizofrénicos con ideas delirantes de grandeza. El consumo de cannabis produce entre los consumidores aumento de síntomas maniformes (Rottanburg y cols, 1982), también entre los sujetos esquizofrénicos (Núñez Domínguez y Gurpegui, datos no publicados).

También podemos recurrir a hallazgos a nivel molecular para explicar los datos encontrados. Martín Calderón y cols (1998) hallaron que, en ratas, los cannabinoides no desplazaban, en un primer momento, a los neurolépticos de su unión a los receptores dopaminérgicos D2 y sí de los D1. Estos hallazgos, sin ser extrapolables a humanos, apoyarían la hipótesis de Knudsen y Vilmar (1984) acerca de la competitividad que muestran el cannabis y los neurolépticos por la unión a los receptores dopaminérgicos. Podríamos suponer que, dado que el cannabis no provoca los efectos esperados por los consumidores cuando están recibiendo tratamiento con neurolépticos atípicos, reducen o abandonan dicho consumo (algo similar a lo que sucede a heroinómanos tratados con naltrexona). Asímismo, si los cannabinoides desplazan a los neurolépticos de la unión a los receptores D1 implicados en la génesis de los síntomas extrapiramidales, los consumidores apreciarían una disminución de dichos efectos secundarios, con el consiguiente refuerzo para el consumo; en el caso de los neurolépticos atípicos, con un perfil de baja unión a receptores D1, dicho refuerzo no se daría. En el grupo AT se ha observado una disminución del consumo en un $40 \%$ de los casos y el abandono del consumo en 4 pacien- tes, junto a un consumo igual en el otro $50 \%$ de los pacientes.

También el cannabis interactúa con otros sistemas en el Sistema Nervioso Central (sistema opioide, colinérgico, serotoninérgico, etc.). Dichas interacciones podrían explicar los efectos psicológicos que su consumo provoca entre sujetos sanos (dopamina-euforia, opioides endógenos-relajación, etc) (Manzanares y cols., 1999), así como entre los esquizofrénicos (dopamina-aumento de sintomatología positiva, acetilcolinatrastornos cognitivos, etc.). El hecho de que también los neurolépticos atípicos muestren un mecanismo de acción más amplio, en el sentido de unión a receptores de distintos sistemas (dopamina, serotonina) podría explicar su mejor efecto en este tipo de esquizofrénicos (por ejemplo, menor incidencia de efectos secundarios extrapiramidales).

Todos estos hallazgos podrían justificar que el consumo de cannabis sea un factor de riesgo entre los esquizofrénicos: aumento de sintomatología, peor cumplimiento terapeútico, ambos indicadores de mala evolución (Núñez Domínguez, 1997). La aplicación de una terapia farmacológica capaz de bloquear la acción de los cannabinoides sería de gran utilidad para el manejo de estos pacientes. Los datos del estudio que, para conocimiento del autor, es el primero de estas características (con pacientes esquizofrénicos consumidores de cannabis) que se ha llevado a cabo, apoyan el uso de los neurolépticos atípicos.

Se podría argumentar que las características que muestran los sujetos del grupo ED son mejores que las del grupo AT ( menor número de ingresos involuntarios). Éstos últimos mostraban de inicio características indicadoras de mayor gravedad del trastorno: más jóvenes, mayor número de ingresos con menor tiempo de consumo y más antecedentes familiares de esquizofrenia. Durante el año de seguimiento observamos en este último grupo cambios con mayor significancia clínica en cuanto al pronóstico: han disminuido el consumo de manera casi significativa con respecto al año previo (en cuatro casos lo han abandonado) y en meyor proporción que el grupo ED, el número de ingresos es similar $(0,65$ para el AT versus $0,36 \%$ en el ED), en comparación con las cifras previas de ingresos antes de empezar el tratamiento con neurolépticos ( 6,85 ingresos de media en el AT versus 3,68 en el ED), con una disminución casi significativa en el número de los ingresos involuntarios durante el último año y no han cambiado el tipo de sustancia de consumo (en el ED 3 sujetos lo han hecho). Y todo ello en el plazo de un año de tratamiento.

El diseño del estudio presenta algunas características que pueden limitar la validez de los resultados, como pueden ser el reducido tamaño y el distinto número de sujetos en las muestras, lo cual puede repercutir en los datos estadísticos, pero los criterios 
de selección fueron estrictos con el fin de delimitar las sustancias consumidas. Es habitual el consumo de diversos tóxicos entre los consumidores, sobre todo de alcohol, lo que aumenta los trastornos provocados por el consumo (Martínez- Arévalo y cols., 1995)

\section{CONCLUSIONES}

Los resultados obtenidos suponen que el uso de neurolépticos atípicos en sujetos esquizofrénicos con uso concomitante de cannabis puede ser de gran utilidad, más aún en sujetos con alto grado de psicopatología, en el sentido de una disminución o abandono del consumo, lo que conlleva un menor riesgo para el paciente y un mejor control de la enfermedad, sobre todo en el aspecto de una mayor conciencia de enfermedad. No podemos olvidar que el tratamiento psiquiátrico que reciben estos pacientes no está basado únicamente en la psicofarmacología, sino que suele estar acompañado de terapias psicológicas de diverso tipo encaminadas, por lo general, a que el paciente adquiera una mayor conciencia de enfermedad $y$, por consiguiente, al abandono del consumo de cannabis $u$ otras sustancias, junto a un mejor cumplimiento de las pautas terapeúticas. El uso de fármacos que faciliten esta tarea supone una gran ayuda para el terapeuta.

La ausencia de trabajos de investigación similares impide una comparación de los resultados, pero los datos obtenidos nos indican que este puede ser un campo de investigación que debe continuar, dado el alto número de pacientes implicados (el cannabis ocupa el primer lugar como sustancia ilegal de consumo por los esquizofrénicos), y cuyo número va en aumento: el informe anual del Observatorio Español sobre Drogas (1999) muestra un incremento en el número de consumidores de cannabis en el último año entre la población; es de suponer que el aumento también se da entre los pacientes psiquiátricos.

\section{BIBLIOGRAFÍA}

Albanese MJ, Bartel RL, Brunno RF, Morgenbesser MW, Schatzberg AF (1994) Comparison of measurements used to determine substance abuse in an impatient psychiatric population. American Journal of Psychiatry. 151: 1077- 1078.

Allebeck P, Adamsson C, Engström A, Rydberg U (1993). Cannabis and schizophrenia: a longitudinal study of cases treated in Stockhlom County. Acta Psychiatrica Scandinavica, 88: 21-24.

Andreasson S, Allebeck P. Engström A, Rydberg U (1987) Cannabis and schizophrenia. A longitudinal study of swedish conscripts. Lancet, ii,: 1483-85.

Beck M, Brook S, Trandafir Al (1999) A comparison of olanzapine with haloperidol an cannabis-induced psychotic disorder: a double-blind randomized controlled trial. Interantional Clinical Psychopharmacology, 14: 177180.

Bowers MB Jr., Mazure CM, Nelson JC, Jatlow (1990) Psychotogenic drug use and neuroleptic response. Schizophrenia Bulletin, 16,1: 81-85.

Buckley PF (1998) Substance abuse in schizophrenia: A review. Journal of Clinical Psychiatry, 59, suppl. 3, 26-30

Buckley P, Thompson P, Way L, Meltzer HY (1994). Substance abuse among partients with treatment-resistant schizophrenia: characteristics and implications for clozapine therapy. American Journal of Psychiatry, 151(3): 385-390.

Camí J (1988). Farmacología de la cannabis. Comunidad y Drogas, 7: 31-60.

Casas M: "Evaluación de la seguridad y tolerancia de risperidona en el tratamiento de pacientes con abuso y dependencia de opiáceos y trastornos psicóticos". XXV Jornadas Nacionales Socidrogalcohol, Tarragona, 23-25 de Abril de 1988

David A, Buchanan A, reed A, Almeida O (1992) The assesment of insight in psychosis. British Journal of Psychiatry, 161: 699-602.

Dixon L, Haas G, Weiden P, Sweeney J, Frances A (1990) Acute effects of drug abuse in schizophrenics patients: clinical observations and patients'self-report. Schizophrenia Bulletin, 18,1: 69-79.

DSM-IV (1995). Manual diagnóstico y estadístico de los trastonos mentales. Masson. Barcelona.

Emrich HM, Leweke M, Schneider U (1997) Towards a cannabinoid hyptothesis of schizophrenia: Cognitive impairments due to dysregulation on the endogenous cannabinoid system. Pharmacology Biochemistry and Behavior, 56, 4: 803- 807

Ghaemi SN, pope HG (1994) Lack of insight in psychotic and affective disorders: a review of empirical studies. Harvard Rev Psychiatry, 2,(1): 22-32.

Hindin R., McCusker J, Vickers-Lathl M, Bigelow C, Garfield F, Lewis B (1994) Radioinmunoessay of hair for determination of cacoine, heroine and marihuana exposure: comparison with self-report. The International Journal of Addiction, 29 (6): 771-789.

Hambrecht M (1999) Cannabis, vulnerability and the onset of schizophrenia: An epidemiological perspective. Inaugural International Cannabis and Psychosis Conference, Melbourne, 16 y 17 de Febrero.

Khantzian EJ (1985) The self-medication hyphotesis of addictive disorders: focus on heroin and cocaine dependence. American Journal of Psychiatry, 142:1259-1264.

Knudsen P, Vilmar T (1984) Cannabis and neuroleptic agents in schizophrenia. Acta Psychiatrica Scandinavica, 69:162-174.

Lin F, Spiga R, Forstach W (1979) Insight and adherence to medication in chronic schizophrencs. Journal of Clinical Psichiatry, 40: 430-2. 
Linzsen DH, Dingemans PM, Lenior ME (1994). Cannabis abuse and the course of recent-onset schizophrenic disorders. Archives of General Psychiatry, 51: 273279.

Manzanares J, Corchero J, Romero J, Fernández-Ruiz JJ, Ramos JA, Fuentes JA (1999) Pharmacological and biochemical interactions between opioids and cannabinoids. Trends in Pharmacological Neurosciences, $20: 287-294$

Martín Calderón JL, Rodríguez de Fonseca F, Muñoz RM, Del Arco I, Villanúa MA, Bilbao A, Escuredo L, Navarro $M$ (1998) Effect of chronic neuroleptic treatment on the acute actions of the cannabinoid agonist HU-210 in rats. 1998 Symposium in The Cannabinoids, Interantional Cannabinoid Research Society, Montpellier (Francia), 23-25 de Julio.

Martínez Arévalo MJ, Calcedo Ordóñez A, Varo Prieto JR, Peralta Rodrigo C (1992) Esquizofrenia, cannabis y conflictos con la ley. Anales de Psiquiatría, 8:9:358-361.

Martínez-Arévalo MJ, Varo Prieto JR, Aguinaga Aguinaga M, Calcedo Ordóñez A (1995) Consumo de cannabis y alcohol como factores de riesgo en la evolución a corto plazo de la esquizofrenia. Actas Luso-Españolas de Neurología y Psiquiatría, 23 (4): 184-192.

McEvoy JP, Freter S, Merritt M, Apperson LJ (1989) Insight about psychosis amohg outpatients with schizophrenia. Hospital \& Community Psychiatry, 44: 883-884.

Meil WM, Schetcher MD (1997) Olanzapine atenuates the reinforcing effects of cocaine. European Journal of Pharmacology, 340 (1): 17-26.

Misra L, Kofoed L (1997) Risperidone in the treatment of metanphetamine psychosis (letter) American Journal of Psychiatry, 154: 1170

Muesser KT, Yarnold PR, Levinson DF, Singh H, Bellack AS, Kee K, Morrison RL, Yadalam KG (1990) Prevalence of substance abuse in schizophrenia: demographic and clinical correlates. Schizophrenia Bulletin, 16:31-56.

Muesser KT, Yarnold PR, Bellack AS (1992) Diagnostic and demographic correlates of substance abuse in schizophrenia and major affective disorder. Acta Psychatrica Scandinavica, 85:48-55.

Negrete JC, Knapp WP, Douglas DE, Smith WB (1986) Cannabis affects the severity of schizophrenic symptoms: results of a clinical review. Psychological Medicine, 16: 000-000.

Noguera Ormazábal B, Mendivil Ferrandez JL, Eguiluz Urruchurtu I, González Torres MA (1993) El doble diagnóstico. Esquizofrenia y toxicomanía: revisión de la literatura. Psiquis, 14:3:119-127.

Núñez Domínguez, LA (1997) Cannabis y psicosis. Relaciones etiopatogénicas. Adicciones, 9, 1: 129-143.
Núñez Domínguez, LA (1998). Cannabis psychosis. A five years follow-up study. 1998 Symposium in The Cannabinoids, Interantional Cannabinoid Research Society, Montpellier (Francia), 23-25 de Julio

Observatorio Español sobre Drogas (1999) Informe $N^{\circ} 2$. Ministerio del Interior, Delegación del Gobierno para el Plan Nacional sobre Drogas, Secretaria Técnica.

Peralta V, Cuesta MJ (1992) Influence of cannabis abuse on schizophrenic psychopathology. Acta Psychiatrica Scandinavica, 85:127-130.

Pristach CA, Smith CM (1990) Medication compliance and substance abuse among schizophrenic patients. Hospital \& Community Psychiatry, 41, 12: 13451348

Regier DA, Farmer ME, Rae DS, Locke BZ, Keith SJ, Judd LL, Goodwin FK (1990) Comorbidity of mental disorders with alcohol and other drug abuse. Results from the Epidemiological Catchment Area (ECA) study. JAMA, 19:2511-2518.

Rottanburg D, Ben-Arie O, Robins AH, Teggin A, Elk A (1982) Cannabis-associated psychosis with hypomanic features. Lancet, i: 1364-1366.

Schneider U, Leweke FM, Mueller-Vahl KR, Emrich HM (1998) Cannabinoid/Anandamide system and schizophenia: Is there evidence for association? Pharmacopsychiatry, 31 (Suppl.), 110-113.

Shaner A, Khlasa ME, Roberts L, Wilkins J, Anglin D, Hsieh SC (1993). Unrecognized cocaine use among schizophrenic patients. American Journal of Psichiatry, 150 (5): 758-762.

Siris SG (1990) Pharmacological treatment of substanceabusing schizophrenic patients. Schizophrenia Bulletin, 16: 111-122.

Smelson DA, Roy A, Roy M (1997) Risperidone disminishes cue-eliciting craving in withdrawn cocaine-dependient patients. Canadian Journal of Psychiatry, 42 (9): 984.

Stone AM, Greenstein RA, Gamble G, McLellan AT (1993) ocaine use by schizophrenic outpatients who receive depot neuroleptic medication. Hospital \& Community Psychiatry, 44: 176-177.

Struve FA, Manno BR, Straumanis JJ (1998) Theta hyperfrontality: An additional quantitative EEG sequela of acute and chronic marihuana (THC) exposure. Comunicación presentada en 1998 Symposium on the Cannabinoids. Montpellier (Francia), 23-25 de Julio

Young DA, Davila R, Scher H (1993). Unawareness of illness and neuropsychological perfomance in chronic schizophrenia. Schizophrenia Research, 10: 117-24.

Yovell Y, Opler LA (1994) Clozpaine reverses cocaine craving in a treatment resistant mentally ill chemical abuser; $A$ case report and a hypothesis. Journal of Nervous and Mental Diseases, 182 (10): 591-592. 
